\title{
The effects of stimulus movement on discrimination learning by rhesus monkeys
}

\author{
PERRY M. NEALIS, HARRY F. HARLOW, and STEPHEN J. SUOMI \\ University of Wisconsin, Madison, Wisconsin 53706
}

\begin{abstract}
Two experiments were conducted with test-sophisticated adult rhesus monkeys to determine effects of differentiating and nondifferentiating movement cues on two-choice discrimination learning. In Experiment 1, 12 monkeys were given initial learning problems followed by intradimensional shift problems with movement- and color-differentiated stimulus cues. Results indicated that original learning progressed most rapidly with movement cues, but that subsequent task-shift performance was comparable for movement- and color-cue problems. There was no evidence that learning with either cue dimension transferred to subsequent problems involving the other cue dimension. In Experiment 2, 14 monkeys were given a series of two-choice discrimination problems in which discriminanda differed in color only. Problems were presented under two conditions: (1) both discriminanda moved synchronously during the choice period or (2) both discriminanda remained stationary. Results indicated that the monkeys learned more rapidly when discriminanda were moving rather than stationary. It was concluded that motion is a dimension of comparable saliency to color, although the psychophysical properties need further clarification. It was hypothesized that stimulus movement enhances discrimination learning through facilitation of the appropriate allocation of attention.
\end{abstract}

Early studies of cue utilization in primate discrimination learning were carried out by Harlow (Harlow, 1945a, 1945b; Meyer \& Harlow, 1948), who convincingly demonstrated that color was a prepotent cue for rhesus monkeys. Draper (1965) and Warren (1953, 1954) expanded the generality of Harlow's findings by employing learning set and transfer paradigms, respectively. In fact, color cues proved so salient for monkeys that D'Amato and Fazzaro (1966) were able to demonstrate that monkeys preferred to respond on the basis of information provided by color cues in contrast to pattern cues.

Comparative psychologists have traditionally employed dimensional cues such as color, form, and size. Studies of visual cognitive functions in nonhuman primates have therefore been restricted because dimensional cues other than color are decidedly less salient than color cues. For example, one can imagine the difficulty encountered in carrying out visual combinedcue paradigms with monkeys when they largely respond to the prepotent cue, that is, color.

Since motion perception appears to be a primary mode of perception in many animals (Richards, 1971), it was hypothesized that differential stimulus movement would provide the monkey with highly salient

This research was supported in part by USPHS Grant MH11894 from the National Institute of Mental Health to the University of Wisconsin Primate Laboratory. The authors wish to thank John W. Davenport for his comments on an earlier draft of this paper. Reprint requests should be addressed to Perry M. Nealis, University of Wisconsin Primate Laboratory, 22 North Charter Street, Madison, Wisconsin 53706. discriminable differences. Experiment 1 was a simple test of that hypothesis. Experiment 2 was designed to determine whether or not stimulus movement affected discrimination learning when such movement was not a differentiating cue.

\section{EXPERIMENT 1}

Two hues that had proved highly discriminable in previous test presentations were chosen for the colorcue problems in this experiment. Two levels of the motion dimension $(0$ and $7 \mathrm{deg} / \mathrm{sec})$ were also selected for the motion-cue problems on the basis of pilot study. Stimulus velocity of $7 \mathrm{deg} / \mathrm{sec}$ was selected because it appears to be an optimal speed for sensing moving foveal objects in both man and monkey (see Richards, 1971).

\section{Method}

Subjects. Twelve test-sophisticated adult rhesus monkeys served as subjects. All subjects had been previously trained to respond by pressing transparent panels that overlaid transilluminated two-dimensional stimuli presented in a DSD display (see Nealis, Carpentier, Suomi, \& Harlow, 1975).

Procedure. Pretraining. Following adaptation to the DSD testing procedure (Nealis et al., 1975), subjects received 100 trials on which a red or green triangular image $(\mathrm{S}+)$ appeared in one of two adjacent display windows as determined by a Gellerman sequence (Gellerman, 1933). At random on half of the trials the S+ was stationary, while on the remaining trials it was moving in either an upward or downward direction with a velocity of $7 \mathrm{deg} / \mathrm{sec}$. This procedure minimized differential reinforcement of responses to particular values of the color and motion dimensions.

Training. The discriminanda for each of four problems were two-dimensional triangular images with surface areas of $7 \mathrm{~cm}^{2}$. 
In Problem $M$ the $S+$ (colored blue) was continuously moving in an upward or downward direction which was counterbalanced over trials. The $S-$ (colored blue) was maintained in a stationary position. Problem $\mathbf{M}^{\prime}$ represented an intradimensional shift task in which the $\mathbf{S}+$ was motionless while the $S-$ was moving. In Problem $C$ the discriminanda remained stationary and were differentiated by color cues, that is, red (S+) vs. green ( $\left.S_{-}\right)$. Problem $C^{\prime}$ was an intradimensional shift task in which the $\mathrm{S}+$ was green and the $S-$ was red. The monkeys were trained for 50 trials/day on each problem to a criterion of $9 / 10$ correct responses (100\% CRF). The problem order for half of the subjects was $M C M^{\prime} C^{\prime}$, while for the remaining six subjects it was $\mathbf{C} \mathbf{M ~ C}^{\prime} \mathbf{M}^{\prime}$.

Data analysis. The trials-to-criterion scores were subjected to square-root transformation due to heterogeneity of variance as indicated by the distribution of raw scores (see Myers, 1966, p. 64). The transformed scores were subjected to analysis of variance with a between-subjects factor of problem order and within-subjects factors of cue dimension (color cues vs. motion cues) and task shift (M and $C$ problems vs. $M^{\prime}$ and $C^{\prime}$ problems). For clarity of report, the original scores were used to compute the mean values illustrated in Figure 1.

\section{Results and Discussion}

The main effect of cue dimensions was statistically significant $(F=6.3, d f=1 / 10, p<.05)$, which reflected the monkeys' ability to reach criterion on problems involving motion-differentiated discriminanda in $38 \%$ fewer trials than on problems involving color-differentiated discriminanda (see Figure 1, left panel). Performance improved with practice on both types of problems

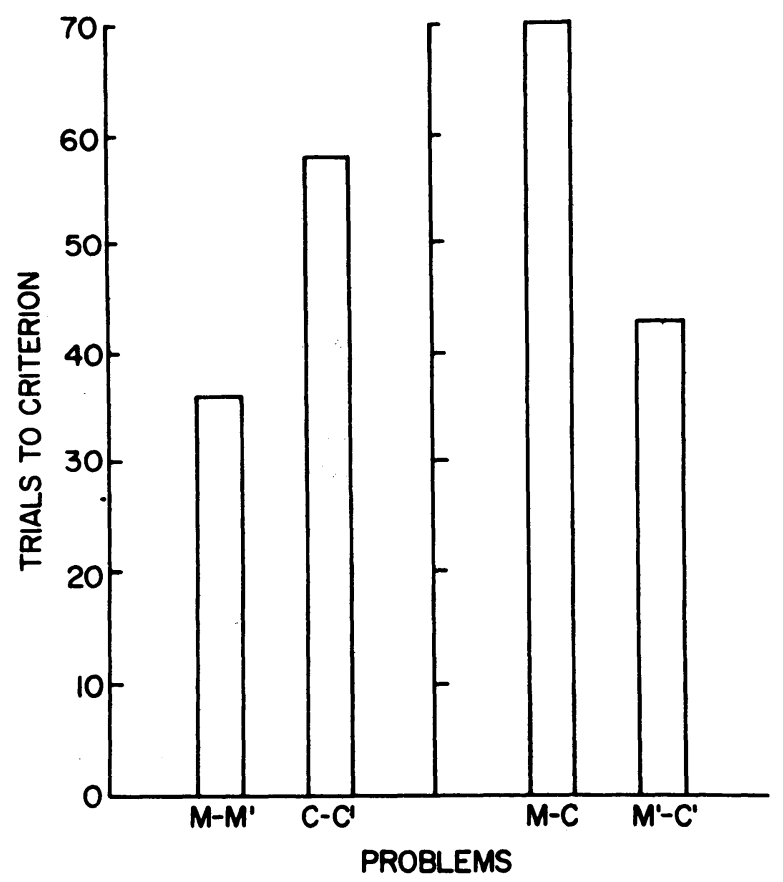

Figure 1. Mean number of trials to criterion on combined motion-cue problems $\left(M, M^{\prime}\right)$ and combined color-cue problems (C, $\left.C^{\prime}\right)$ from Experiment 1 are shown in the left panel; mean number of trials to criterion on the original learning problems combined $(M, C)$ and the intradimensional shift problems combined $\left(M^{\prime}, C^{\prime}\right)$ from Experiment 1 are shown in right panel. (see Figure 1, right panel). Subjects reached criterion on the intradimensional shift tasks $\left(M^{\prime}\right.$ and $\left.C^{\prime}\right)$ in approximately half the number of trials that were required to reach criterion on the original learning tasks, $\mathrm{M}$ and $\mathrm{C}$ $(\mathrm{F}=11.0, \mathrm{df}=1 / 10, \mathrm{p}<.01)$.

There was no evidence of differential practice effects since the interaction of Cue Dimension by Task Shift was nonsignificant $(F=.4)$. Additionally, differential specific transference between problems was absent, as indicated by a lack of statistical significance for all terms involving problem order (all ps $>.10$ ). It appears, then, that the monkeys more rapidly acquired the motion-cue discriminations in contrast to the color-cue discriminations, but that rapid solution of the initial motion-cue problem did not result in more rapid solution of the subsequent color-cue problem. Similarly, there was no evidence of general positive transfer from the first color-cue problem to the first motion-cue problem.

\section{EXPERIMENT 2}

In this experiment rhesus monkey performance on color-cue discrimination problems was contrasted under two conditions: (1) when both discriminanda remained stationary and (2) when both discriminanda moved synchronously during the choice period. Hence, in contrast to the first experiment, stimulus movement provided no differentiating cues in this experiment.

\section{Method}

Subjects. Ten monkeys that had served in the first experiment and four additional test-sophisticated adult rhesus monkeys were randomly assigned to two equal groups, Group M and Group S. The four DSD-naive monkeys were adapted to the test apparatus in the usual manner.

Procedure. Pretraining. Subjects received 100 trials on each of two random figure discrimination problems on 2 days immediately preceding training.

Training. The subjects received 24 trials on a single twochoice color discrimination problem on each of 8 consecutive days. The differentiating color cues were different for the eight problems. Both groups of monkeys received identical problems in the same order of presentation. However, the discriminanda for Group $M$ always moved in an upward direction with a constant velocity of $7 \mathrm{deg} / \mathrm{sec}$, while for Group $S$ the discriminanda remained stationary.

Data analysis. The number of correct responses was subjected to analysis of variance with one between-subjects factor of groups (moving vs. stationary discriminanda) and one withinsubjects factor of problems (1-8).

\section{Results and Discussion}

Performance significantly improved for both groups of monkeys over the series of eight problems $(F=6.2$, $\mathrm{df}=7 / 84, \mathrm{p}<.05)$, though the groups differed significantly in their rates of improvement $(\mathrm{F}=2.1, \mathrm{df}=7 / 84$, $\mathrm{p}<.05)$. Pairwise comparisons of group means for each of the eight problems by Fisher's 1.s.d. statistic at the 5\% confidence level (see Fryer, 1966) revealed that Group $M$ initially reached significantly higher per- 


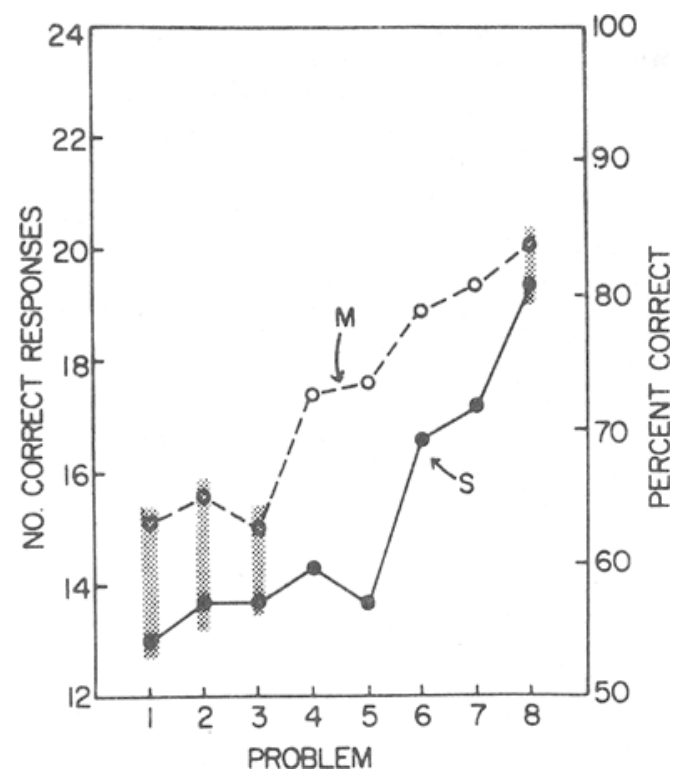

Figure 2. Mean number of correct responses on color discrimination problems when discriminanda are moving (M) vs. stationary (S) as a function of the problem series in Experiment 2 (means that do not differ at the $5 \%$ confidence level are shaded).

formance levels than Group S (see Figure 2). Inspection of the learning curves indicated that subjects reached their highest performance levels in the problem series approximately 40-50 trials earlier when both discriminanda were moving than when both discriminanda were stationary.

It was interesting to note that superior performance on the moving-discriminanda problems did not appear until the monkeys had encountered the fourth problem in the series, that is, on the 4th day of testing. This finding suggests that the long-term memory components involved in this type of learning are somehow altered by conditions of stimulus movement. It appears that attentional processes could act as mediating factors in the development of such long-term memory components. This point will be addressed in the following discussion.

\section{GENERAL DISCUSSION}

The results of Experiment 1 suggested that monkeys acquire two-choice discriminations more rapidly when the discriminanda are differentiated along a dimension of movement in contrast to a dimension of color. However, caution must be exercised in extrapolating from these data because only two values of each cue dimension were employed in Experiment 1. There may exist certain hues that provide monkeys with discriminable differences comparable to those provided by motion cues. There may also exist degrees of relative movement that provide monkeys with discriminable differences equal to certain color cues.

Another interpretive problem arises from the results of the first experiment. Do performance differences between motioncue and color-cue discrimination problems remain constant with continued practice? The analysis of such temporal effects might provide clues as to the nature of those differences. In earlier investigations of cue saliency (e.g., Draper, 1965; Meyer \& Harlow, 1948; Warren, 1953), consistent performance differences between color discriminations and form (or size) discriminations persisted even through the development of learning sets. Those findings suggest that the underlying learning processes reflect a permanency that might characterize the processes accountable for learning differences observed under conditions of motion- vs. color-differentiating cues. Instead, the results of Experiment 2 suggest that superior discrimination learning performance on motion-cue problems may be attributed to a temporary rather than permanent change in stimulus processing.

The facilitation of color discrimination learning that is brought about when the discriminanda are moving represents an effect that does not fit well with certain theoretical accounts of cue saliency (e.g., Archer, 1962; Trabasso, 1963). Trabasso (1963) has suggested that certain cues can act as "emphasizers"; for example, an arrow that points to the critical cue may increase the speed of concept identification learning in children. However, it seems unlikely that movement serves to emphasize differential color cues in a similar fashion since the discriminanda in Experiment 2 differed only in color; that is, there were no irrelevant cues that would otherwise compete for attention. Moreover, the prepotency of color cues should have precluded the necessity of further emphasis (Meyer \& Harlow, 1948). It, therefore, seems unlikely that movement acts as an emphasizer of cues in color discrimination learning. Archer (1962) has proposed an "obviousness-of-cues" hypothesis, suggesting that discriminable differences between stimuli can be increased through the addition of other salient differentiating cues. The obviousness-of-cues hypothesis fails, however, to explain the facilitation of learning found in Experiment 2 because movement was not a differentiating cue in those problems. Hence, it appears that moving discriminanda enhance learning independently of the obviousness of color cues.

In summary, it appears that stimulus movement can serve two functions in nonhuman primate learning tasks. First, the differential movement of discriminanda constitutes a powerful discriminative cue for rhesus monkeys. It seems likely that motion cues are at least as salient as color cues in discrimination learning. Second, the nondifferential movement of discriminanda may facilitate discrimination learning because movement enhances the appropriate allocation of attention. This facilitation effect is particularly impressive because it can be demonstrated in learning situations that already provide the monkey with prepotent cues, that is, color cues. Finally, an attractive feature of motion cues is that generally they do not alter the information conveyed by dimensional cues such as size or shape because movement constitutes a stimulus state rather than a stimulus quality.

\section{REFERENCES}

Archer, E. J. Concept identification as a function of obviousness of relevant and irrelevant information. Journal of Experimental Psychology, 1962, 63, 616-620.

DRAPER, W. A. Cue dominance in oddity discriminations by rhesus monkeys. Journal of Comparative and Physiological Psychology, 1965, 60, 140-141.

D'Amato, M. R., \& Fazzaro, J. Attention and cueproducing behavior in the monkey. Journal of the Experimental Analysis of Behavior, 1966, 9, 469-473.

FrYer, H. C. Concepts and methods of experimental statistics. Boston: Allyn \& Bacon, 1966. Pp. 259-271.

Gellerman, R. W. Chance orders of alternating stimuli in visual discrimination experiments. Journal of Genetic Psychology, 1933, 42, 206-207.

HaRlow, H. F. Studies in discrimination learning by monkeys: V. Initial performance by experimentally naive monkeys on stimulus-object and pattern discriminations. Journal of Genetic Psychology, 1945, 33, 3-10. (a) 
HaRlow, H. F. Studies in discrimination learning by monkeys: VI. Discrimination between stimuli differing in both color and form. only in color, and only in form. Journal of Genetic Psychology, 1945, 33, 225-235. (b)

MeYer, D. R., \& Harlow, H. F. The development of transfer of responses to patterning by monkeys. Journal of Comparative and Physiological Psychology, 1948, 42, 454-464.

MyERS. J. L. Fundamentals of experimental design. Boston: Allyn \& Bacon, 1966.

Nealis, P. M., Carpentier, A. M., Suomi, S. J., \& HaRlow. H. F. Dynamic scimulus display for the WGTA. Behavior Research Methods \& Instrumentation, 1975, 7, 291-293.
RichaRds, W. Motion detection in man and other animals. Brain Behavior and Evolution, 1971, 4, 162-181.

Trabasso, T. Stimulus emphasis and all-or-none learning of concept identification. Journal of Experimental Psychology, 1963, 65, 395-406.

WARREN, J. M. Additivity of cues in visual pattern discrimination by monkeys. Journal of Comparative and Physiological Psychology, 1953, 46, 484-488.

WARREN, J. M. Perceptual dominance in discrimination learning in monkeys. Journal of Comparative and Physiological Psychology, 1954, 47, 290-292.

(Received for publication June 12, 1977.) 